\title{
Diselenide Crosslinks for Enhanced and Simplified Oxidative Protein Folding
}

\author{
Reem Mousa, ${ }^{[1]}$ Taghreed Hidmi, ${ }^{[1]}$ Sergei Pomyalov, ${ }^{[1]}$ Shifra Lansky, ${ }^{[1]}$ Lareen \\ Khouri, ${ }^{[1]}$ Deborah E. Shalev, ${ }^{[2,3]}$ Gil Shoham ${ }^{[1]^{*}}$, and Norman Metanis ${ }^{[1]^{*}}$ \\ ${ }^{[1]}$ Institute of Chemistry, The Hebrew University of Jerusalem, Edmond J. Safra, Givat Ram, \\ Jerusalem 9190401; ${ }^{[2]}$ Department of Pharmaceutical Engineering, Azrieli College of \\ Engineering Jerusalem; and ${ }^{[3]}$ Wolfson Centre for Applied Structural Biology, The Hebrew \\ University of Jerusalem, Edmond J. Safra, Givat Ram, Jerusalem 9190401 (Israel) \\ E-mail: gil2@mail.huji.ac.il and Metanis@mail.huji.ac.il
}

\begin{abstract}
The oxidative folding of proteins has been studied for over sixty years, providing critical insight into protein folding mechanisms. Hirudin, the most potent natural inhibitor of thrombin, is a 65-residue protein with three disulfide bonds, and is viewed as a folding model for a wide range of disulfide-rich proteins. Hirudin's folding pathway is plagued with highly heterogeneous intermediates and scrambled isomers, affecting its folding rate and yield. Aiming to overcome this limitation, we introduced diselenide bridges at native and non-native positions and investigated their effect on hirudin's folding, structure and activity. Our studies demonstrated that, regardless of the specific positions of these substitutions, the diselenide crosslinks enhanced the folding rate and yield of the corresponding hirudin analogs, while reducing the complexity and heterogeneity of the process. Moreover, crystal structural analysis confirmed that the diselenide substations maintained the overall structure of the protein without causing major changes in function. The current choice of hirudin as a study model has implications beyond its specific folding mechanism, demonstrating the high potential of diselenide substitutions in the designing, preparation and characterization of disulfide-rich proteins.
\end{abstract}




\section{Introduction}

The in vitro oxidative folding of many proteins is usually a rather sluggish process, making it often a major bottleneck in the preparation of therapeutic, disulfide-rich proteins. ${ }^{[1]}$ During this relatively slow process, a reduced/unfolded polypeptide undergoes conformational folding combined with disulfide bond intermediates formation, eventually reaching the native state $(\mathrm{N})$ of the protein with its final disulfide crosslinks. ${ }^{[2]}$ Despite the disadvantage, the slow kinetics of disulfide bond formation and shuffling enable chemical trapping, isolation and further characterization of the folding intermediates, ${ }^{[2 a, 3]}$ allowing an important in-depth elucidation of the folding process. Extensive studies of such processes have shown divergent folding pathways that differ mainly in the heterogeneity of the intermediates. ${ }^{[2]}$ At the ends of this wide spectrum of processes, there are two extreme mechanism models, represented by bovine pancreatic trypsin inhibitor (BPTI) and hirudin. ${ }^{[2]}$ In one extreme there is the bifurcated folding mechanism of BPTI, which is characterized by the predominance of a finite number of distinct intermediates. These intermediates have native-like structures, which assume one, and then two, native disulfide bonds, before reaching the final conformational state of the protein with its three native disulfide bonds. ${ }^{[4]}$ The other extreme is hirudin, a small protein isolated from the leech Hirudo medicinalis ${ }^{[5]}$, which is known to be the most potent natural inhibitor of thrombin. ${ }^{[6]}$ Hirudin contains two major parts, a compact N-terminal domain, held by three disulfide crosslinks, and an extended, practically unstructured, C-terminal peptide (Fig. 1a). ${ }^{[7]}$ Unlike BPTI, the folding pathway of hirudin involves numerous, highly heterogeneous disulfide-bonded intermediates, generally known as a "trial and error" mechanism. ${ }^{[2 \mathrm{~b}, 8]}$ Reduced hirudin folds into its final native state in two stages; first, hirudin undergoes a non-specific packing stage, with random disulfide pairing, to form the heterogeneous intermediate ensembles, involving a single disulfide (1-SS), two disulfides (2-SS) and three disulfides (3-SS) scrambled species (Fig. 1b). The second, and rate-determining, stage is the disulfide reshuffling of the heterogeneous scrambled population, leading to the formation of the final structure with its three native disulfide bonds, 6-14, 16-28 and 22-39 (Fig. 1a). ${ }^{[2 b, 8]}$

Scrambled isomer formation and the accumulation of trapped intermediates are known to decrease the efficiency of in vitro oxidative folding of many proteins, thereby limiting their folding rate and yield. ${ }^{[9]}$ One of the potential approaches to overcome 
such limitations involves the use of selenium-containing molecules, which can act as inter/intramolecular catalysts in disulfide exchange reactions. ${ }^{[10]} \mathrm{A}$ similar approach involves cysteine to selenocysteine ( $\mathrm{Sec}, \mathrm{U}$ ) substitutions, which has been used to study the oxidative folding of several proteins, ${ }^{[10 \mathrm{e}, 11]}$ as was done in the isosteric replacement of native and non-native disulfides with diselenides in BPTI. ${ }^{[1 \mathrm{~g}, 12]}$ All the Seleno-BPTI (Se-BPTI) analogs were found to fold correctly into the native state, some of which were demonstrated to bypass the common trapped intermediates of the folding pathway. ${ }^{[12 b]}$ These improved folding results of the Se-BPTI case study, motivated us to investigate the effect of diselenide substitution on the folding pathway of hirudin, the opposite folding model. Given the more chaotic folding pathway of hirudin, we speculated that the introduction of diselenide crosslinks within the hirudin protein would decrease the distribution of folding intermediates, and thereby improving the overall folding rate. That is, in minimizing the random disulfide parings inherent to the native folding pathway, it was expected that the productive folding routes will be enhanced over the unproductive ones, leading to a more efficient folding process.

\section{Results and discussion}

In the present work, following the above rationale, we prepared and characterized four seleno-hirudin (Se-Hir) analogs in order to study the effect of selenocysteine substitutions on the folding, the structure and the biological activity of the protein. Three of the analogs contained diselenides at the native crosslinks, 6-14, 16-28 and 22-39, while the fourth analog introduced a diselenide bond at a non-native crosslink, 6-16. Overall, our results demonstrate that disulfide-to-diselenide substitutions of the native disulfide crosslinks improve the folding efficiency to the native state, significantly minimizing the formation of non-productive intermediates. Remarkably, this was also the case with the non-native diselenide-containing analog 6-16. Two of the Se-Hir analogs studied here indicated a simpler folding pathway, where the common 1-SS intermediate ensembles were not observed (vide infra). Interestingly, the overall 3D structure of the Se-Hir analogs remained almost unchanged, as confirmed unequivocally by crystal structure studies of the corresponding Se-Hir-thrombin complexes. Two of the analogs exhibited a slightly lower inhibitory activity, probably 
as a result of small local conformational changes around the substituted selenium atoms, mainly at positions 6,14 and/or 16 .

The current study began with the full chemical synthesis of wild-type hirudin (WT-Hir) and the four Se-Hir analogs described above. All five proteins were chemically prepared from two segments and a single native chemical ligation $(\mathrm{NCL})^{[13]}$ reaction at the Gln38-Cys39 junction (Scheme 1). ${ }^{[14]}$ The corresponding C-terminal peptides Hir(39-65), containing Cys/Sec39, were synthesized by Fmoc-SPPS, ${ }^{[15]}$ purified and characterized by HPLC and ESI-MS (Fig. S1 and S2). Similarly prepared by the FmocSPPS approach, were the Hir(1-38)-COSR N-terminal peptides, bearing a C-terminal thioester surrogate, ${ }^{[16]}$ and $\mathrm{Sec}$ at different specific positions $(6,14,16,22$ and/or 28) as needed (for details see SI, and Fig. S3-S7). A Cys/Sec-NCL ${ }^{[17]}$ was then performed for the preparation of all the analogs studied (Fig. S8-S12), providing milligram quantities of the homogenous proteins. For the Se-Hir analogs this resulted in proteins with a single diselenide crosslink and four reduced thiols, while the WT-Hir protein was isolated in the fully reduced form, as confirmed by MS analysis.

Once the proteins have been synthesized and purified, they were subjected to oxidative folding under anaerobic conditions at $\mathrm{pH}$ 8.7, using oxidized glutathione (GSSG) as the oxidant (see details in the SI). ${ }^{[\mathrm{b}]}$ For WT-Hir, under these conditions, the common three ensemble populations 1-SS, 2-SS, and 3-SS, could be clearly observed during the folding process. These species overlapped extensively and hence appeared in the HPLC output as broad peaks (Fig. 2a), in a good agreement with previous reports. ${ }^{[8 b, 18]}$ As folding proceeded, and within $0.5 \mathrm{~h}$, the 3 -SS species predominated and remained stable even after $1.5 \mathrm{~h}^{\left[{ }^{[9,19]}\right.}$ The final stage of reshuffling ended only after $7 \mathrm{~h}$, yielding about $88 \%$ of the native form of WT-Hir (Table 1, Fig. 2a, Fig. S20).

For the Se-Hir analog Hir(C16U/C28U), a significantly faster folding process was observed under the same conditions, as compared to WT-Hir. More than half of the protein reached the native state within $0.5 \mathrm{~h}$, and the entire folding process was completed in $1.5 \mathrm{~h}$, yielding about $90 \%$ of the native form of the protein (Table 1, Fig. $2 b$, Fig. S21c). The intermediates of this process appeared as fewer and more distinct peaks, containing mainly 2- and 3-crosslinks. This pattern suggests a significant decrease in the heterogeneous populations (1-SS, 2-SS and 3-SS), where scrambled intermediates predominate and rearranged more rapidly to the final native state. Thus, 
the Sec substitution at positions 16 and 28 lead to about 5-fold increase in the folding rate relative to WT-Hir, with comparable overall yield, and in good correlation with the initial predictions made above. The advantage of using Sec in oxidative folding is even more significant under aerobic conditions and in the absence of additional oxidants. ${ }^{[11 \mathrm{f} \text {, }}$ 12b] Air oxidation of $\operatorname{Hir}(\mathrm{C} 16 \mathrm{U} / \mathrm{C} 28 \mathrm{U})$ resulted in $80 \%$ overall yield of folded protein after $5 \mathrm{~h}$, and these results remained unchanged even after $22 \mathrm{~h}$. In comparison, under the same conditions, the folding of WT-Hir resulted in only $20 \%$ overall yield of folded protein (Fig. 3a and S13). These results also emphasize the importance of the GSSG component in oxidative protein folding, which appears to be critical not only as an oxidant but also as a catalyst for the disulfide (and diselenide) reshuffling. ${ }^{[2]}$ In this respect it is noted that although the Sec substitution apparently steers the folding process to form higher ratios of productive intermediates, a small number of trapped unproductive intermediates could be observed alongside the main process, which appear to be stable even after $7 \mathrm{~h}$. This indicates that scrambled isomers in this Se-Hir analog react differently towards reshuffling, ${ }^{[9]}$ and a small fraction become even more stable as a result of the Cys to Sec substitutions.

The Se-Hir analog Hir(C22U/C39U) also showed a significant improvement of its folding efficiency under anaerobic conditions. About $66 \%$ of the protein reached the native state within $5 \mathrm{~min}$, and the entire folding process was completed in $1.5 \mathrm{~h}$, yielding about $94 \%$ of the native form of the protein (Table 1). Interestingly, a major HPLC peak was detected at 23 min, which contain mainly the 3-SS intermediate species (Fig. 2c, Fig. S21d).

Although the folding yield of the $\operatorname{Hir}(\mathrm{C} 6 \mathrm{U} / \mathrm{C} 14 \mathrm{U})$ was similar to the other proteins (Table 1, Fig. 2d, and Fig. S21b), this analog formed significantly fewer intermediate peaks during the folding process (Fig. 2d). These results are in good correlation with previous kinetic and computational studies, which indicated that not all disulfide crosslinks have a similar role during the folding process of hirudin. The 6-14 crosslink was proposed to play a critical role, since it was found to be highly populated among the 1-SS, 2-SS, and 3-SS species in WT-Hir. ${ }^{[9,20]}$ Unlike WT-Hir, Hir(C16U/C28U) and Hir(C22U/C39U), no 1-SS species were detected during the folding process of Hir(C6U/C14U) (Fig. 2d). This can be explained by a pre-existing 6-14 diselenide crosslink, which, once present, is likely to more easily govern the formation of the 
remaining disulfide bonds. Thus, these current results further confirm the critical role of the 6-14 disulfide bond in the hirudin folding pathway (Fig. 1b).

Despite its favorable folding rate and yield, we found that the Hir(C6U/C14U) analog displays a lower inhibitory activity toward its natural target thrombin. The inhibitory constant $K_{I}$ of this analog for thrombin was about 20 -fold higher than either WT-Hir ${ }^{[6 b \text {, }}$ ${ }^{21]}$ or the other seleno-analogs, $\operatorname{Hir}(\mathrm{C} 16 \mathrm{U} / \mathrm{C} 28 \mathrm{U})$ and $\operatorname{Hir}(\mathrm{C} 22 \mathrm{U} / \mathrm{C} 39 \mathrm{U})$ (Fig. 3b, Table 1). Additionally, a distinct difference was observed in the retention time of the folded form of the $\mathrm{Hir}(\mathrm{C} 6 \mathrm{U} / \mathrm{C} 14 \mathrm{U})$ analog, as compared to the other seleno-variants (Fig. 2f). Although a similar observations have been reported by Alewood et al. of the selenoanalogs of conotoxins, ${ }^{[25]}$ the differences we observed in the retention time combined with the lower activity against thrombin raised our concern at to whether the Hir(C6U/C14U) analog is correctly folded into the native state.

These relatively small differences motivated us to examine more closely the detailed 3D structures of the studied proteins. Since free hirudin proteins proved to be quite challenging to crystallize and analyze, we undertook a comprehensive structural analysis of the relevant hirudin analogs using the crystal structure of their bound complexes with thrombin. ${ }^{[7,22]}$ The three Se-Hir analogs described above were incubated with bovine thrombin to form the corresponding complexes, namely Hir(C16U/C28U)-thrombin (Complex-1), Hir(C6U/C14U)-thrombin (Complex-2) and Hir(C22U/C39U)-thrombin (Complex-3). The complexes were crystallized and their detailed 3D structures were determined and analyzed (see details in the SI). In general, all three structures were found to be quite similar to each other, and to the reported 3D structure of WT-Hir with bovine thrombin (PDB: 1HRT, Fig. 1a). ${ }^{[23]}$ A global structural comparison of the bound Se-Hir in Complexes 1-3, to the bound WT-Hir in this reference structure (1HRT) gives relatively small (overall) RMSD values of 1.620 , $1.476,1.472 \AA$, respectively. These values, as well as a visual superposition of each of these pairs (Fig. 4) indeed demonstrate that no major global structural differences could be observed between the four structures compared here. This result is important, as it confirms unequivocally that despite the replacement of a disulfide bond with a diselenide bond at different positions; all three Se-Hir analogs achieved the native folded state, with practically the same 3D structure. This conclusion is further supported by spectral analysis of the free $\operatorname{Hir}(\mathrm{C} 6 \mathrm{U} / \mathrm{C} 14 \mathrm{U})$ analog in solution, which 
was confirmed by $2 \mathrm{D}{ }^{1} \mathrm{H}-\mathrm{NMR}$ COSY to have an overall chemical shift pattern that agrees with that of the natural wild-type protein (Fig. S19).

A closer look at the Se-Hir analogs within these complexes, and in comparison to WTHir, confirmed that most of the intramolecular hydrogen bonds have been closely maintained, especially the tight cluster of H-bonds located generally around the 6-14 disulfide (or diselenide) crosslink. A superposition of the Se-Hir structures in Complex1 and Complex-2 (Table S3 in SI), demonstrates that this central H-bonding cluster remains practically unchanged, and emphasizes its central role in the stabilization of the core structure of hirudin. Especially critical within this cluster are the interactions made by Lys47, which play a key role in holding the hirudin N-terminal and C-terminal peptides in their specific directions. These results reinforce previous studies which showed that the hydrogen bonds formed by the sidechain amino group of Lys47 with the backbone carbonyl of Asp5 and the hydroxyl sidechain of Thr4 help to position the $\mathrm{N}$-terminal of hirudin in the active-site cleft of thrombin. ${ }^{[22]}$ Interestingly, only small local conformational differences are apparent around the three original disulfide bonds that have been substituted with diselenides, perhaps as the result of changes in the bond length, dihedral angles and atom size (Fig. S15). These local changes appear to be most critical in the case of the $\operatorname{Hir}(\mathrm{C} 6 \mathrm{U} / \mathrm{C} 14 \mathrm{U})$ analog, probably due to the critical location of the 6-14 crosslink within the central H-bonding cluster (Table S2). This was further supported by $2 \mathrm{D}{ }^{1} \mathrm{H}-\mathrm{NMR}$ COSY, in which deviations in chemical shift by NMR were observed in the region of Thr4, Gln11, Leu13, Leu15 and Cys16.

Along the rationale outlined above, it was interesting to investigate how a non-native diselenide crosslink influence the hirudin folding process. Previous studies on apamin and BPTI, where non-native diselenide substitutions were introduced, showed contradictory results. While a non-native diselenide substitution impeded the formation of the native species in the short polypeptide apamin; ${ }^{[11 \mathrm{~b}]}$ a BPTI analog folded perfectly into its native state with two selenylsulfides and one disulfide crosslinks. ${ }^{[12 b]}$ In the case of hirudin, we selected the non-native crosslink 6-16 due to its predominance in 1-SS and 2-SS ensembles (vide supra). ${ }^{[20]}$ Our experiment demonstrated that the Hir(C6U/C16U) analog folded in a similar pathway to WT-Hir, forming a heterogeneous mixture of species, mainly the 2-SS and 3-SS intermediates, that rapidly converted into the native state with a similar yield to WT-Hir (Table 1, Fig. 2e, and Fig. S21e). This analog, similar to Hir(C6U/C14U), showed a 10-fold reduction in its 
inhibitory activity toward thrombin (Table 1, Fig. 3b), supporting our previous interpretation that a fine-tuned structural perturbation around $\mathrm{Cys} / \mathrm{Sec}$ at 6,14 and/or 16 may be responsible for the decreased activity observed for these two analogs (vide supra).

\section{Conclusions}

In summary, we studied the effect of diselenide substitutions on the folding of the model protein hirudin. We show that a regioselective diselenide crosslink formation reduces the complexity and heterogeneity of the folding pathway of hirudin and thereby enhances the folding rate and its efficiency. Interestingly, in the case of the native 6-14 and non-native 6-16 diselenide substitutions, the 1-SS populations were mostly absent, showing that the early-stages of hirudin folding could be bypassed entirely, ${ }^{[9,20]}$ and yet to result in the native state. However, these two diselenide substitutions slightly reduced the inhibitory activity of the corresponding hirudin analogs, likely due to subtle structural perturbations surrounding the Cys/Sec6,14 and/or 16 residues. Fortunately, the substitution of the other two native crosslinks with diselenides had practically no effect on the 3D structure and inhibitory activity of these Se-Hir analogs, yet still demonstrating the general folding improvement observed for all the Se-Hir analogs. These results indicate that although disulfide-to-diselenide substitution proved to be generally advantageous for the preparation of hirudin analogs, and other proteins, the exact location of the replaced crosslink could be important for a full retention of its inhibitory activity.

The choice of hirudin as the present model study seems to have implications beyond its specific folding mechanism. Unlike BPTI, the defining characteristic of hirudin lies in the complex route it follows to achieve its native fold, and this seemingly chaotic pathway is shared by many other proteins. ${ }^{[2]}$ Furthermore, many proteins adopt a folding mechanism that lies somewhere on the spectrum between these two opposite models. ${ }^{[2 b]} \mathrm{We}$ found that the substitution of native/non-native disulfide with diselenide bonds typically enhances protein folding and yield, mostly without any major changes in the protein structure, and some with no significant effect on function. Based on these results, the current study of the seleno-hirudin analogs extends the previous studies on selenocysteine-containing analogs of BPTI, ${ }^{[12 b, 12 c]}$ conotoxins, ${ }^{[11 \mathrm{c}-\mathrm{f}]}$ and insulin, ${ }^{[24]}$ 
which together demonstrate the high potential of disulfide-to-diselenide substitutions in basic and applied research. In addition to its obvious advantage in the study and preparation of disulfide-rich proteins, such an approach may also prove useful for a wide range of applications, including the rational improvement of the stability, activity and specificity of this unique family of proteins.

\section{Acknowledgements}

We thank Mrs. Ricki Notis Dardashti for input on the manuscript. N.M. acknowledges the financial support of Israel Science Foundation (1072/14 and 783/18) and ICRF Acceleration Grant. G. S. acknowledges the support of both the Israel Science Foundation (1905/15) and The Israeli Ministry of Science (3-12484/15). R. M. acknowledges the support of the VATAT scholarship for Arab students. T.H. is grateful to the Neubauer Fellowship for Arab Ph.D. students. 


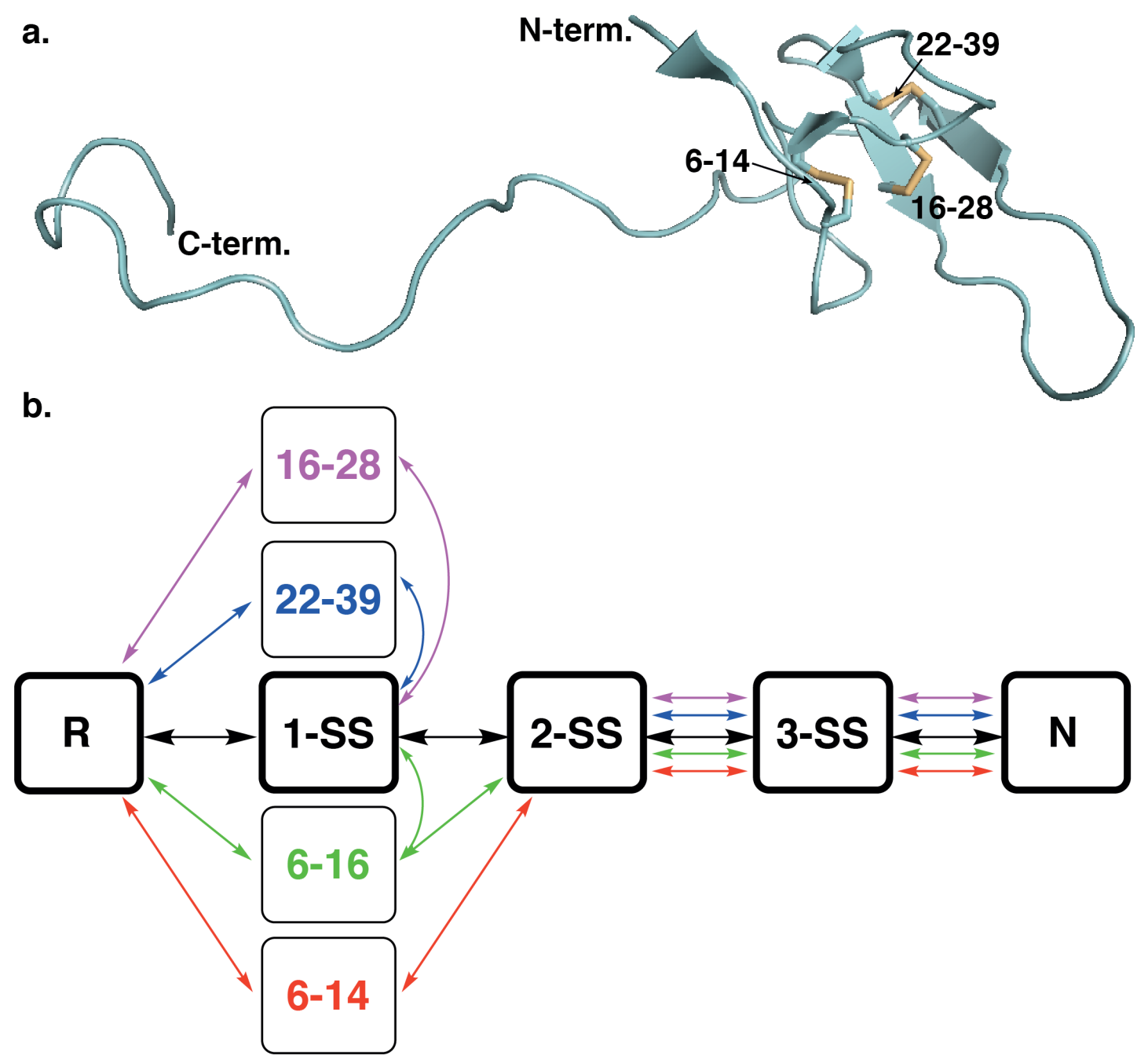

Figure 1. 3D Structure and oxidative folding mechanism of hirudin. a. 3D structure of hirudin (PDB entry: 1HRT) based on crystal structure of its complex with bovine thrombin, which is not shown here. The three native disulfide bonds 16-28, 6-14, and 22-39 are shown as sticks (yellow); b. the proposed oxidative folding mechanism of WT-Hir (in black) and its seleno-analogs (colored). ${ }^{[12,13]} \mathrm{R}$ and $\mathrm{N}$ are reduced/unfolded and native hirudin, respectively; 1-SS, 2-SS and 3-SS are ensembles of molecules with the corresponding number of disulfide bonds. The four synthetic Se-Hir analogs (colored) contain a diselenide bond at the specified positions. All Se-Hir analogs folded faster than WT-Hir, and with fewer intermediates. Although all analogs formed the 2SS and 3-SS ensembles, the 1-SS populations were mostly absent in $\operatorname{Hir}(\mathrm{C} 6 \mathrm{U} / \mathrm{C} 14 \mathrm{U})$ and $\operatorname{Hir}(\mathrm{C} 6 \mathrm{U} / \mathrm{C} 16 \mathrm{U})$. 


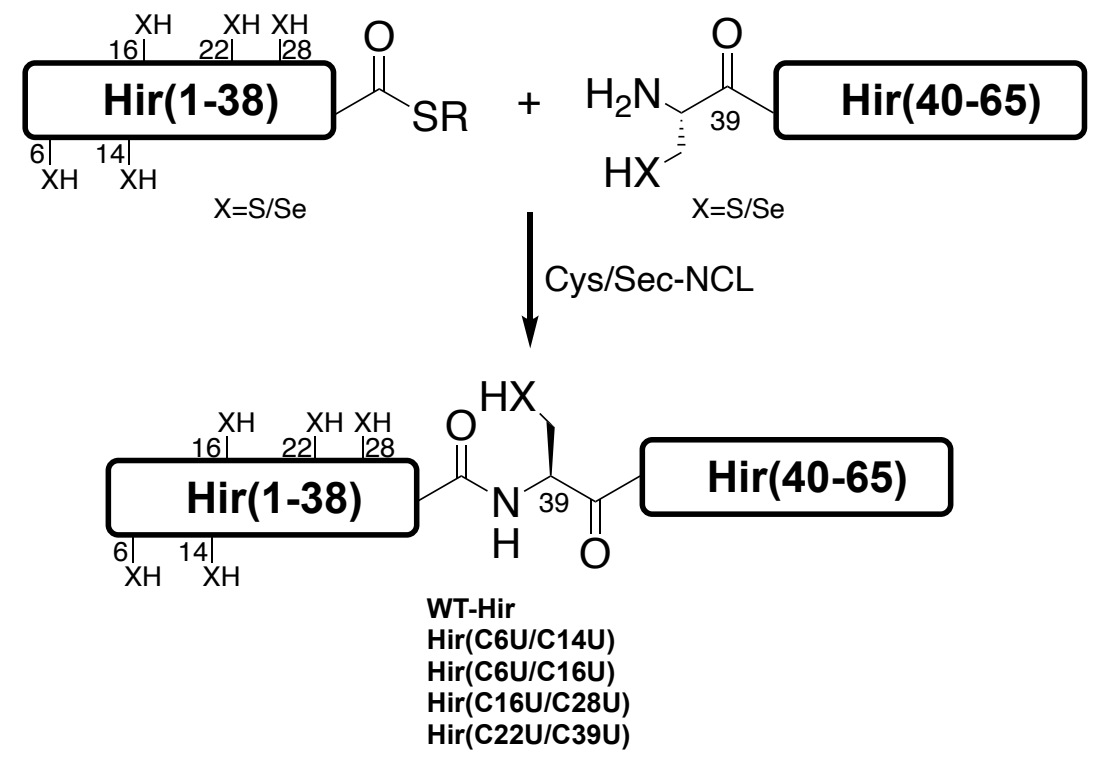

Scheme 1. Chemical synthesis of WT-Hir and its seleno-analogs; Hir(C6U/C14U), Hir(C6U/C16U), Hir(C16U/C28U), and Hir(C22U/C39U) by native chemical ligation $(\mathrm{NCL})$ reactions. 

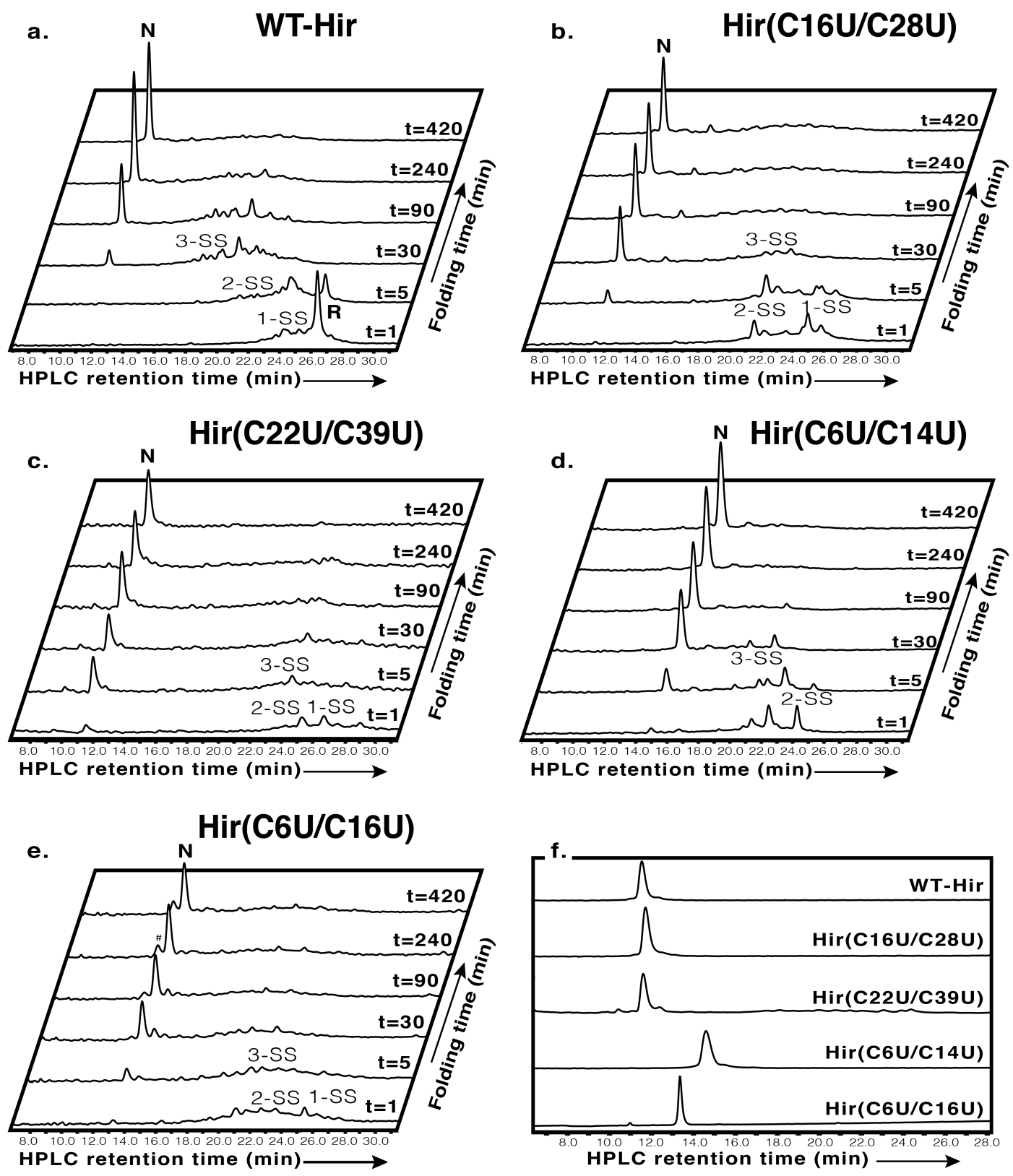

Figure 2. The anaerobic oxidative folding at $\mathrm{pH} 8.7$ in the presence of $150 \mu \mathrm{M}$ GSSG and $30 \mu \mathrm{M}$ of (a) WT-Hir; (b) $\mathrm{Hir}(\mathrm{C} 16 \mathrm{U} / \mathrm{C} 28 \mathrm{U})$; (c) $\mathrm{Hir}(\mathrm{C} 22 \mathrm{U} / \mathrm{C} 39 \mathrm{U})$; (d) Hir(C6U/C14U); and (e) Hir(C6U/C16U). \# an impurity with unknown mass. 1-SS, 2SS and 3-SS represents the number of disulfide (or diselenide) crosslinks in the intermediates. (f) Retention times of isolated native states of WT-Hir and the selenovariants. 
Table 1. Characterization of WT-Hir and the Se-Hir analogs.

\begin{tabular}{|c|c|c|c|}
\hline Protein & Yield\% by HPLC (isolated) & HPLC r.t. of N@ $(\min )$ & $K_{\mathrm{I}}(\mathrm{pM})^{\#}$ \\
\hline WT-Hir & $88 \%(61 \pm 1 \%)$ & 11.2 & $10.9 \pm 4.9$ \\
\hline Hir(C16U/C28U) & $90 \%(56 \pm 1 \%)$ & 11.3 & $10.0 \pm 3.7$ \\
\hline Hir(C22U/C39U) & $94 \%(61 \pm 1 \%)$ & 11.3 & $12.5 \pm 2.9$ \\
\hline Hir(C6U/C14U) & $95 \%(66 \pm 1 \%)$ & 14.8 & $192.4 \pm 21.9$ \\
\hline Hir(C6U/C16U) & $90 \%(46 \pm 1 \%)$ & 13.2 & $104.9 \pm 15.0$ \\
\hline
\end{tabular}

@ the retention time (r.t.) of the native state $(\mathrm{N})$ is shown in Fig. 2f. ${ }^{\#}$ The inhibitory activity of the different hirudin analogs with Thrombin was assayed in buffer at $37^{\circ} \mathrm{C}$ and using $\mathrm{N}$-( $p$-Tosyl)-Gly-Pro-Arg- $p$-nitroanilide as a substrate, where the initial rate of $p$-nitroaniline formation was followed at $405 \mathrm{~nm}$ by UV (complete details in SI). 


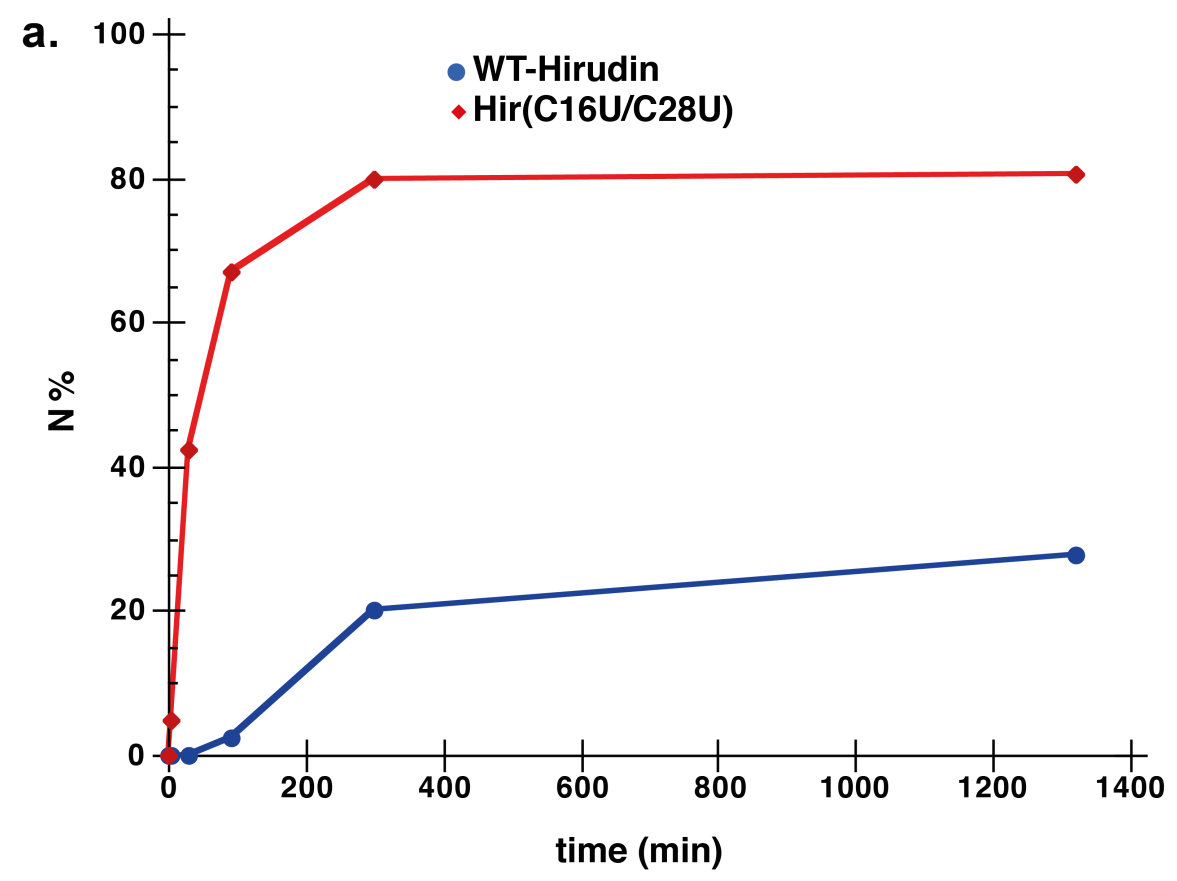

b.

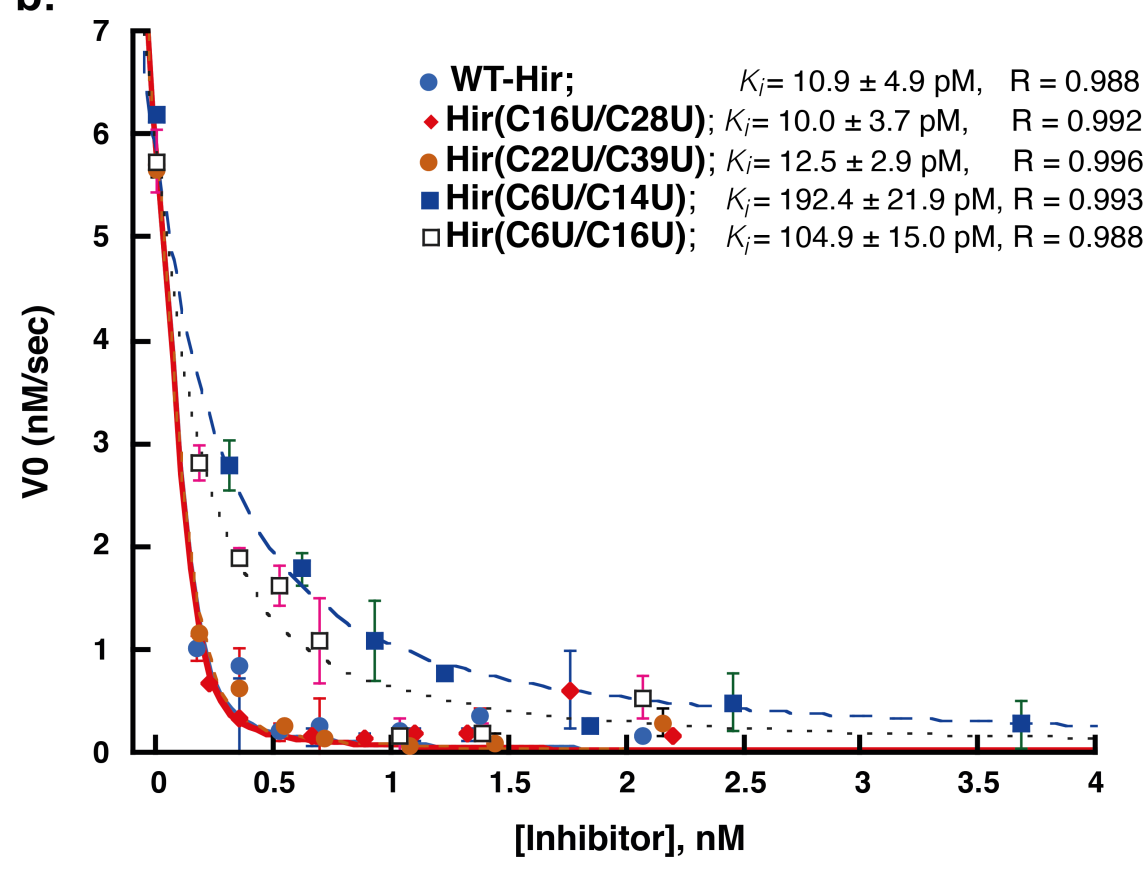

Figure 3. a. Kinetic traces of the aerobic folding in the absence of GSSG and with 30 $\mu \mathrm{M}$ WT-Hir or Hir(C16U/C28U) at $\mathrm{pH}$ 8.7. The lines connecting the data points are shown only for illustrative purposes and do not represent a data fit. b. Inhibition activity of WT-Hir and Se-Hir analogs. The inhibition of bovine thrombin by WT-Hir (blue circles), Hir(C16U/C28U) (red diamonds), Hir(C22U/C39U) (brown circles), $\operatorname{Hir}(\mathrm{C} 6 \mathrm{U} / \mathrm{C} 14 \mathrm{U})$ (blue filled squares), and Hir(C6U/C16U) (open squares). The data were fitted as described in the Supporting Information to give the apparent $K_{\mathrm{I}}$ values indicated. 


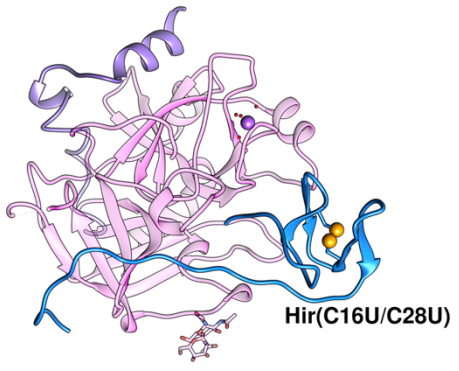

a

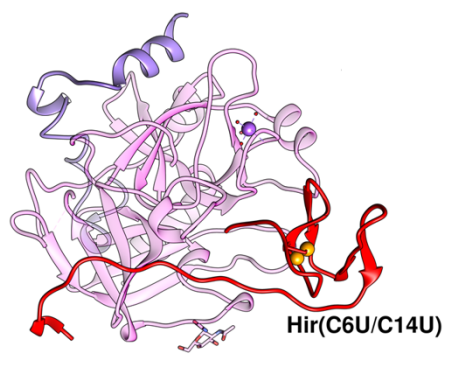

b

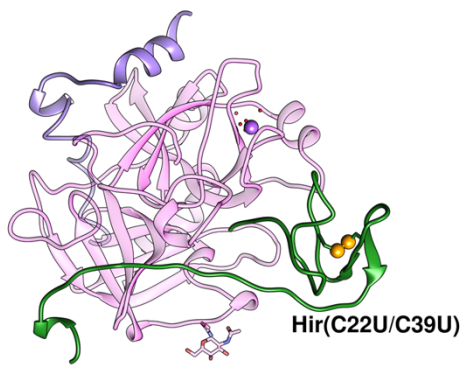

Figure 4. The overall structures of the three Se-Hir-Thrombin complexes. a. Complex1; b. Complex-2; c. Complex-3. All complexes are shown in the same orientation, where the thrombin active site is in the bottom-right, opening to the right. Color codes: Thrombin heavy chain (dark purple); Thrombin light chain (light purple); Hir(C16U/C28U) (blue); Hir(C6U/C14U) (red); Hir(C22U/C39U) (green). Gold spheres represent the Se-Se bonds. 


\section{Bibliography}

[1] a) B. J. Tombling, C. N. K. Wang, D. J. Craik, Angew. Chem. Int. Ed. 2020, 59, 11218-11232;

b) M. Gongora-Benitez, J. Tulla-Puche, F. Albericio, Chem Rev 2014, 114, 901-926.

[2] a) B. S. Mainathambika, J. C. Bardwell, Annu Rev Cell Dev Bi 2008, 24, 211-235; b) J. Y. Chang, Biochemistry 2011, 50, 3414-3431; c) J. L. Arolas, F. X. Aviles, J. Y. Chang, S. Ventura, Trends Biochem. Sci. 2006, 31, 292-301.

[3] C. B. Anfinsen, Science 1973, 181, 223-230.

[4] a) T. E. Creighton, Prog. Biophys. Mol. Biol. 1978, 33, 231-297; b) J. S. Weissman, P. S. Kim, Science 1991, 253, 1386-1393; c) R. Mousa, S. Lansky, G. Shoham, N. Metanis, Chem. Sci. 2018, 9, 4814-4820.

[5] J. Dodt, H. P. Muller, U. Seemuller, J. Y. Chang, Febs Lett. 1984, 165, 180-184.

[6] a) C. G. Binnie, B. W. Erickson, J. Hermans, Febs Lett. 1990, 270, 85-89; b) S. R. Stone, J. Hofsteenge, Biochemistry 1986, 25, 4622-4628.

[7] T. J. Rydel, A. Tulinsky, W. Bode, R. Huber, J. Mol. Biol. 1991, 221, 583-601.

[8] a) B. Chatrenet, J. Y. Chang, J. Biol. Chem. 1992, 267, 3038-3043; b) B. Chatrenet, J. Y. Chang, J. Biol. Chem. 1993, 268, 20988-20996.

[9] J. Y. Chang, J. Biol. Chem. 1995, 270, 25661-25666.

[10] a) J. Beld, K. J. Woycechowsky, D. Hilvert, Biochemistry 2007, 46, 5382-5390; b) J. Beld, K. J. Woycechowsky, D. Hilvert, Biochemistry 2008, 47, 6985-6987; c) J. Beld, K. J. Woycechowsky, D. Hilvert, Biochemistry 2009, 48, 4662-4662; d) R. J. Hondal, S. M. Marino, V. N. Gladyshev, Antioxid. Redox Signal. 2013, 18, 1675-1689; e) R. Mousa, R. N. Dardashti, N. Metanis, Angew. Chem. Int. Ed. 2017, 56, 15818-15827; f) R. Mousa, P. S. Reddy, N. Metanis, Synlett 2017, 28, 1389-1393; g) P. S. Reddy, N. Metanis, Chem. Commun. 2016, 52, 3336-3339; h) N. Metanis, C. Foletti, J. Beld, D. Hilvert, Isr. J. Chem. 2011, 51, 953-959.

[11] a) S. Pegoraro, S. Fiori, S. Rudolph-Bohner, T. X. Watanabe, L. Moroder, J. Mol. Biol. 1998, 284, 779-792; b) S. Pegoraro, S. Fiori, J. Cramer, S. Rudolph-Bohner, L. Moroder, Prot. Sci. 1999, 8, 1605-1613; c) M. Muttenthaler, S. T. Nevin, A. A. Grishin, S. T. Ngo, P. T. Choy, N. L. Daly, S. H. Hu, C. J. Armishaw, C. I. A. Wang, R. J. Lewis, J. L. Martin, P. G. Noakes, D. J. Craik, D. J. Adams, P. F. Alewood, J. Am. Chem. Soc. 2010, 132, 3514-3522; d) A. D. de Araujo, B. Callaghan, S. T. Nevin, N. L. Daly, D. J. Craik, M. Moretta, G. Hopping, M. J. Christie, D. J. Adams, P. F. Alewood, Angew. Chem. Int. Ed. 2011, 50, 6527-6529; e) K. H. Gowd, V. Yarotskyy, K. S. Elmslie, J. J. Skalicky, B. M. Olivera, G. Bulaj, Biochemistry 2010, 49, 2741-2752; f) A. M. Steiner, K. J. Woycechowsky, B. M. Olivera, G. Bulaj, Angew. Chem. Int. Ed. 2012, 51, 5580-5584; g) N. Metanis, D. Hilvert, Curr. Opin. Chem. Biol. 2014, 22, 27-34.

[12] a) N. Metanis, J. Beld, D. Hilvert, Patai's Chemistry of Functional Groups 2011; b) N. Metanis, D. Hilvert, Angew. Chem. Int. Ed. 2012, 51, 5585-5588; c) N. Metanis, D. Hilvert, Chem. Sci. 2015, 6, 322-325.

[13] a) P. E. Dawson, T. W. Muir, I. Clark-Lewis, S. B. H. Kent, Science 1994, 266, 776-779; b) S. B. H. Kent, Chem. Soc. Rev. 2009, 38, 338-351.

[14] The chemical synthesis of Hirudin-P6 variant as well as the Thr43 O-glycosylated and Tyr61 sulfated analogs were previously reported by Payne and coworkers, using the Lys27-Cys28 ligation junction. Y. S. Y. Hsieh, L. C. Wijeyewickrema, B. L. Wilkinson, R. N. Pike, R. J. Payne, Angew. Chem. Int. Ed. 2014, 53, 3947-3951.

[15] a) R. B. Merrifield, J. Am. Chem. Soc. 1963, 85, 2149-2154; b) R. Behrendt, P. White, J. Offer, J. Pept. Sci. 2016, 22, 4-27.

[16] a) J. B. Blanco-Canosa, P. E. Dawson, Angew. Chem. Int. Ed. 2008, 47, 6851-6855; b) D. T. Flood, J. C. J. Hintzen, M. J. Bird, P. A. Cistrone, J. S. Chen, P. E. Dawson, Angew Chem. Int. Ed. 2018, 57, 11634-11639. 
[17] a) P. S. Reddy, S. Dery, N. Metanis, Angew. Chem. Int. Ed. 2016, 55, 992-995; b) R. J. Hondal, B. L. Nilsson, R. T. Raines, J. Am. Chem. Soc. 2001, 123, 5140-5141; c) M. D. Gieselman, L. Xie, W. A. van der Donk, Org. Lett. 2001, 3, 1331-1334; d) R. Quaderer, A. Sewing, D. Hilvert, Helv. Chim. Acta 2001, 84, 1197-1206.

[18] J. Y. Chang, Biochem. J. 1995, 300, 643-650.

[19] J. Y. Chang, P. Schindler, B. Chatrenet, J. Biol. Chem. 1995, 270, 11992-11997.

[20] C. Micheletti, V. De Filippis, A. Maritan, F. Seno, Proteins 2003, 53, 720-730.

[21] A. Otto, R. Seckler, Eur. J. Biochem. 1991, 202, 67-73.

[22] T. J. Rydel, K. G. Ravichandran, A. Tulinsky, W. Bode, R. Huber, C. Roitsch, J. W. Fenton, Science 1990, 249, 277-280.

[23] J. Vitali, P. D. Martin, M. G. Malkowski, W. D. Robertson, J. B. Lazar, R. C. Winant, P. H. Johnson, B. F. P. Edwards, J. Biol. Chem. 1992, 267, 17670-17678.

[24] a) O. Weil-Ktorza, N. Rege, S. Lansky, D. E. Shalev, G. Shoham, M. A. Weiss, N. Metanis, Chem-Eur. J. 2019, 25, 8513-8521; b) B. Dhayalan, Y. S. Chen, N. B. Phillips, M. Swain, N. K. Rege, A. Mirsalehi, M. Jarosinski, F. Ismail-Beigi, N. Metanis, M. A. Weiss, Chem-Eur. J. 2020, 26, 4695-4700; c) K. Arai, T. Takei, M. Okumura, S. Watanabe, Y. Amagai, Y. Asahina, L. Moroder, H. Hojo, K. Inaba, M. Iwaoka, Angew Chem. Int. Ed. 2017, 56, 5522-5526. 